\title{
Quality of Life-Spasticity-Fatigue Score in Patients With Multiple Sclerosis and Serum D Vitamin Level
}

\author{
Yasemin Ozkan
}

\begin{abstract}
Background: Vitamin D (VitD) is being increasingly studied in multiple sclerosis (MS). Association of VitD deficiency and MS risk, progression, disability and severity has been shown in previous studies. Our objective was to prospectively investigate if 25 -hydroxyvitamin $\mathrm{D}(25(\mathrm{OH}) \mathrm{D})$ levels are associated with duration of disease, the number of joint involvement, level of spasticity, and quality of life (QoL) in MS.
\end{abstract}

Methods: The study was performed on 40 patients who fulfilled the Poser criteria for primary progressive MS (PPMS), repeater MS (RRMS) or diagnosed with secondary progressive MS (SPMS). Serum samples of all participants were collected for 25(OH)D measurement. 25(OH)D levels were divided into three groups: VitD deficient (serum level below $20 \mathrm{ng} / \mathrm{mL}$ ), VitD insufficient (serum level between 21 and $29 \mathrm{ng} / \mathrm{mL}$ ), and normal VitD (serum level higher than $30 \mathrm{ng} / \mathrm{mL}$ ). Demographic characteristics and medical history of the patients were recorded. Modified Ashworth scale was used to assess spasticity. As a measure of QoL, the MS international quality of life (MUSIQOL) questionnaire and MS fatigue scores were used.

Results: We found that VitD levels in patients with attack type MS were significantly lower than the primary and progressive MS types. In addition, serum VitD level was associated with the level of the disability and the duration of disease. According to the results of our study, there is a significant relationship between mean VitD levels and the mean score of spasticity and also of MS fatigue scores.

Conclusion: VitD levels are associated with duration of disease, the number of joint involvement, level of spasticity, and QoL in MS.

Keywords: Multiple sclerosis; Spasticity; Quality of life; Fatigue scores

\section{Introduction}

Multiple sclerosis (MS) causes functional loss in different

Manuscript accepted for publication January 28, 2016

Physical Medicine and Rehabilitation, Department of Medicine, University of Dumlupinar, 4300 Kutahya, Turkey. Email: ftrjo@hotmail.com

doi: http://dx.doi.org/10.14740/jnr372e areas of central nervous system (CNS), and various clinical findings with considerable social and economic impact. The pathogenic mechanisms of MS are not fully understood. MS has been considered to be a disease of autoimmune origin and partly related to genetic and environmental factors (geographic and sociocultural factors, nutritional habits and other factors). The most prevalent age of MS is between 20 and 40 years, and it is more common among women than men. Experimental studies supported the view that vitamin D (VitD) might have a role in MS pathogenesis. There is epidemiological evidence that the risk of developing MS is increased in association with low VitD status. Recent studies indicate that 25-hydroxyvitamin D $(25(\mathrm{OH}) \mathrm{D})$ levels are lower in MS patients than healthy subjects. In addition, many studies note lower 25OHD levels in more severe disease.

$\mathrm{MS}$ is a chronic demyelinating disease of the CNS. Although its etiology has not been clarified, MS has been considered to be a disease of autoimmune origin and partly related to genetic and environmental factors. Inflammation, demyelination and glial scarring (sclerosis) develop in MS in nerve fibers myelin in CNS white matter in multiple spaces and correspondingly neurological dysfunctions emerge from related systems $[1,2]$.

VitD is involved in regulation of immunity functions, acquired immunity and acquiring the natural immunity. The relation between deficiency and MS development has been shown in the epidemiological studies during the recent years. Experimental studies supported the view that VitD might have a role in MS pathogenesis. Receptors of VitD can be found in many organs and tissues such as $\mathrm{T}$ lymphocytes, brain, prostate, pancreas, gonads, breast tissue, muscle, and colon. Nearly all gene expression studies on VitD have pointed out that active VitD regulates the $0.8-5 \%$ of the total genomes directly or indirectly [3]. This case explains why active VitD has many roles such as regulation of cellular growth, DNA repair, differentiation, apoptosis, membrane transport, cellular metabolism, adhesion, and oxidative stress [4-6]. VitD deficiency decreases proinflammatory cytokines in patients with MS but increases the secretion of anti-inflammatory cytokine. It has been shown that VitD is neuroprotective on brain cells in vitro. This effect of VitD is attributed to the increase of regulation of neuronal $\mathrm{Ca}$, immune modulation, anti-oxidative mechanism, nerve conduction, and detoxification mechanisms $[7,8]$.

The aim of this study was to search the relation between serum VitD level of patients with MS and MS duration of disease, level of spasticity, MS quality of life (QoL), and fatigue scores. 
Table 1. Demographic Data

\begin{tabular}{lll}
\hline Age & $35.85 \pm 7.89$ & $18-53$ \\
Gender & $\mathrm{n}$ & $\%$ \\
$\quad$ Male & 15 & 37.5 \\
$\quad$ Female & 25 & 62.5 \\
\hline
\end{tabular}

\section{Materials and Methods}

This study was performed in the Clinics of the Department of Physical Therapy and Rehabilitation at Kutahya Hospital of Dumlupinar University College of Medicine. All data were collected during the summer of 2015. A total of 40 patients were included in the study. The subjects fulfilled either the Poser criteria for primary progressive MS (PPMS), repeater MS (RRMS) or diagnosed with secondary progressive MS (SPMS). The ages of the patients were between 18 and 65 years. The patients had the auxiliary/independent free-standing ability and EDSS scores were $\leq 6.5$. The patients with metabolic bone disease were not included in the study. Demographic characteristics and medical history of the patients such as age, gender, body mass index (BMI), marital status, education level, occupation, use of assistive devices, type of MS, and the year of the disease were recorded. Modified Ashworth scale was used to assess spasticity. This scale classifies muscle tone from 0 (normal) to 4 (severe spasticity). As a measure of QoL, the MS International Quality of Life (MUSIQOL) questionnaire, a disease-specific QoL scale, was used. Low scores for QoL indicate high levels in QoL. Serum levels of active VitD of patients were examined by the method of enzyme immunoassay analysis. The patients were divided into three groups based on their serum $25(\mathrm{OH}) \mathrm{D}$ levels (normal, insufficient, and deficient).

\section{Statistical analysis}

For statistical analysis, the number and percentage in statistical comparisons, Pearson correlation, analysis of variance and $t$-test were used. Dunnett $C$ test was used as a further test of variance analysis. $\mathrm{P}<0.05$ was considered statistically significant. Data were analyzed using SPSS 18.00 package program.

\section{Results}

A total of $40 \mathrm{MS}$ patients were included in the study. The demographic and clinical characteristics of all participants are summarized in Table 1. Mean age was $35.85 \pm 7.89$ years. Most of the participants $(62.5 \%$ of MS patients) were female. All MS patients were using interferon-beta (INF-beta) as their DMT.
Table 2. Various Features Associated With MS Patients

\begin{tabular}{|c|c|c|}
\hline & Mean \pm SD & Min. - Max. \\
\hline MS year & $6.52 \pm 3.1$ & $1-14$ \\
\hline Vitamin D levels & $13.08 \pm 9$ & $2-34$ \\
\hline \multirow[t]{2}{*}{ Fatigue score } & $3.87 \pm 0.6$ & $2.4-5.1$ \\
\hline & $\mathrm{n}$ & $\%$ \\
\hline \multicolumn{3}{|l|}{ Joint involvement } \\
\hline 1 & 15 & 37.5 \\
\hline 2 & 19 & 47.5 \\
\hline 3 & 5 & 12.5 \\
\hline 4 & 1 & 2.5 \\
\hline \multicolumn{3}{|l|}{ Vitamin D deficient } \\
\hline Serious deficient & 22 & 55.0 \\
\hline Medium deficient & 13 & 32.5 \\
\hline Normal & 5 & 12.5 \\
\hline \multicolumn{3}{|l|}{ MS type } \\
\hline Primary & 17 & 42.5 \\
\hline Attack & 13 & 32.5 \\
\hline Progressive & 10 & 25.0 \\
\hline \multicolumn{3}{|l|}{ Spasm } \\
\hline No & 5 & 12.5 \\
\hline Light & 17 & 42.5 \\
\hline Medium & 12 & 30.0 \\
\hline High & 1 & 2.5 \\
\hline $\begin{array}{l}\text { Serious highly } \\
\text { advanced }\end{array}$ & 3 & 7.5 \\
\hline
\end{tabular}

No additional medications affecting serum VitD were used by patients.

The mean duration of disease in this study group was 6.52 \pm 3.1 years. Most of the participants $(42.5 \%)$ were primary MS. The mean serum VitD level was $13.08 \pm 9 \mathrm{ng} / \mathrm{mL}$ (range 2 - 34 $\mathrm{ng} / \mathrm{mL}$ ) in patients with MS and $55 \%$ of MS patients had significantly lower VitD levels. Nineteen of the patients $(47.5 \%$ of MS patients) had joint involvement score of 2 (Table 2 ).

Seventeen of the patients ( $42.5 \%$ of MS patients) had light degree of spasticity and their QoL rating score was $63.80 \pm$ 10.44 , while their mean walking score was $29.75 \pm 14.9$ (range $12-60$ ) and the mean fatigue score was $3.87 \pm 0.6$ (range 2.4 $-5.1)$.

\section{Statistical comparison}

As shown in Table 3, a statistically significant correlation was

Table 3. The Relationship Between the Level of Vitamin D and Various Parameters

\begin{tabular}{llllll}
\hline & BMI & Duration of MS & Quality of life score & Walking score & Fatigue score \\
\hline VitD level & $\mathrm{r}=-0.0361$ & $\mathrm{r}=-0.783$ & $\mathrm{r}=0.634$ & $\mathrm{r}=-0.763$ & $\mathrm{r}=-0.755$ \\
& $\mathrm{P}=0.022$ & $\mathrm{P}=0.00$ & $\mathrm{P}=0.00$ & $\mathrm{P}=0.00$ & $\mathrm{P}=0.00$ \\
\hline
\end{tabular}


Table 4. Vitamin D Levels in Different BMI Groups

\begin{tabular}{lllll}
\hline VKI & $\begin{array}{l}\text { Number of } \\
\text { subjects }\end{array}$ & Mean & F value & P value \\
\hline Normal weight & 13 & 16.00 & & \\
Overweight & 23 & 13.26 & 4.012 & 0.026 \\
Obese & 4 & 2.50 & & \\
\hline
\end{tabular}

found between VitD level and BMI, the duration of MS, QoL score, and fatigue score. As VitD level increased, VKI, the duration of MS, walking score, and fatigue score decreased and the score on the QoL increased $(\mathrm{P}<0.05)$. Among these parameters, a medium level of correlation existed between VitD level and VKI, while a medium to high level correlation existed between the other variables.

As seen in Table 4, VitD level is statistically significant compared to VKI groups. In the advanced analysis conducted to determine which VKI group the difference stemmed from, it was determined that obese patients have significantly lower VitD level than normal and overweight patients $(\mathrm{P}<0.05)$.

As shown in Table 5, significant changes are noticed in the mean VitD levels as the number of joint involved changed. In the advanced analysis, it was determined that the patients with one joint involvement have significantly higher level of VitD than those with three joint involvements $(\mathrm{P}<0.05)$.

Table 6 shows that VitD level changes significantly between patients with different scores of spasticity. Advanced analysis determined that the individuals without spasticity have significantly higher VitD level than those with mild, medium, serious, and advanced spasticity $(\mathrm{P}<0.05)$.

As shown in Table 7, the mean level of VitD is changed significantly in various patients groups with different MS types. It is noticed that patients with attack type MS have significantly more VitD in serum than those with primary and progressive MS types $(\mathrm{P}<0.05)$.

\section{Discussion}

Findings of the current study suggested that VitD levels in patients with attack type MS were significantly lower than the primary and progressive MS types. In addition, serum VitD level was associated with disability and duration of disease, as had previously been reported, and was significantly related with disability. According to the results of our study, as long as VitD level increases, spasticity and MS fatigue scores decrease.

Table 5. Vitamin D Levels in Patients With Different Joint Involvements

\begin{tabular}{lllll}
\hline $\begin{array}{l}\text { Joint } \\
\text { involvement }\end{array}$ & $\begin{array}{l}\text { Number of } \\
\text { patients }\end{array}$ & Mean & F value & P value \\
\hline 1 & 15 & 17.20 & & \\
2 & 19 & 12.42 & 5.029 & 0.012 \\
$3+$ & 6 & 4.83 & & \\
\hline
\end{tabular}

Table 6. Vitamin D Levels According to Spasticity Scores

\begin{tabular}{lllll}
\hline Spasticity & $\begin{array}{l}\text { Number of } \\
\text { patients }\end{array}$ & Mean & F value & P value \\
\hline Without spasticity & 5 & 31.40 & & \\
Mild & 17 & 12.53 & & \\
Medium & 12 & 10.25 & 20.446 & 0.000 \\
Serious & 4 & 6.50 & & \\
Advanced & 2 & 2.00 & & \\
\hline
\end{tabular}

Findings of study had some similarities to and differences from what have been reported in the literatures to date. Findings of the current study suggested that VitD level was significantly related to duration of disease [9]. VitD deficiency in patients with MS may be due to less exposure to sunlight because of their illness rather than a less exposure to sunlight relationship.

Additional studies reported in our study can be explained with prolonged disease duration and higher disease activity (disability) [9].

They might be expected to have less outdoor activities than normal population; therefore, they are at risk of VitD deficiency in the long term because of poor sunlight exposure [10].

Depending on the literature, we especially included patients with EDSS $\geq 6.5$ in our study assuming that prolonged disease duration and higher disease activity (disability) are affected by duration and frequency of sunlight exposure at the lowest level.

Previous clinical studies, however, were conducted among patients with variable disease durations and could not determine whether low VitD is a consequence of MS activity [11], or whether VitD levels early in the disease course contribute to predicting long-term progression and disability. Because the prevalence of VitD insufficiency $(25(\mathrm{OH}) \mathrm{D}<50 \mathrm{nmol} / \mathrm{L}(20$ $\mathrm{ng} / \mathrm{mL})$ ) is high, supplementation could potentially benefit a large proportion of MS patients [12].

\section{Disability}

There are probably many other factors that might influence MS occurrence and disability. According to the results of our study, VitD deficiency influences greater percent of disability burden among MS patients. Many studies reported a negative correlation between serum VitD levels and disability [13-17].

In their large prospective investigation, Ascherio et al found that average serum 25(OH)D levels in the first 12

Table 7. Vitamin D Levels According to MS Type

\begin{tabular}{lllll}
\hline MS type & $\begin{array}{l}\text { Number of } \\
\text { patients }\end{array}$ & Mean & F value & P value \\
\hline Primary & 17 & 6.29 & & \\
Attack & 13 & 22.23 & 27.369 & 0.000 \\
Progressive & 10 & 12.70 & & \\
\hline
\end{tabular}


months following a CIS strongly predicted MS activity and progression over the subsequent 4 years. By the end of the follow-up at 60 months, those patients with serum 25(OH)D concentrations $\geq 50 \mathrm{nmol} / \mathrm{L}$ had a four times lower change in T2 lesion volume, a two-fold lower rate of brain atrophy, and lower disability than those below $50 \mathrm{nmol} / \mathrm{L}$ [18].

Additional studies reported that higher 25(OH)D levels predicted reduced MS relapse activity, a lower rate of progression and lower disability $[19,20]$.

Additionally, the study by Smolders et al, performed on 267 MS patents, also reported a significant association between lower serum concentration of 25-dihydroxyvitamin D and higher EDSS [21].

In their study conducted on 78 Iranian individuals with RRMS in 2012, Amini Harandi et al found inverse correlation between serum vitamin concentration and EDSS score only in women [22].

Van der Mei and colleagues studied 136 MS patients with higher disability (EDSS $>3$ ) in Australia and found a significantly lower prevalence of VitD insufficiency in MS cases than the control. Among MS cases, increasing disability was strongly associated with lower levels of $25(\mathrm{OH}) \mathrm{D}$ and with reduced sun exposure [23].

Contrary to some studies, in which the levels of VitD were not significantly lower in case group than healthy subjects, our findings revealed a significant difference in 25-dihydroxyvitamin D levels between MS patients compared to control group.

Hamidreza et al from Guilan University of Medical Sciences in northern Iran could not find a significant difference between the VitD levels of a total of 52 patients of MS (EDSS score less than 5.5) and the control. This might be due to the season the study was conducted in or the elevation the study was conducted at [24].

However, Yildiz et al demonstrated no significant difference in serum VitD and EDSS between Swiss MS patients with high and low disease activity [25].

Low sample size in their study and differences in adjusted covariates, skin type variation, geographic situation, socioeconomic status, and different genetic backgrounds in various study populations might explain these different results.

\section{Spasticity}

Spasticity is one of the most prevalent symptoms in MS and the one most affecting the QoL and functionality of patient $[26,27]$, but its pathophysiology is complex and has not been fully understood as noted in various review articles [28]. According to the results of our study, as long as VitD level increases, spasticity decreases. The significant correlation that was determined with serum VitD level in patients with MS can be associated with physical and functional consequences of mechanisms that VitD took part in and social and cognitive consequences of the disease.

Epidemiological studies already indicated that spasticity may affect up to $80 \%$ of patients with MS and that was an important factor contributing to disability in this population [29].

The study by the Multiple Sclerosis International Foundation, an online survey of 692 patients with MS, showed that
$75 \%$ of the survey respondents experienced spasticity in both legs accompanied by main symptoms of stiffness or tension in $88.7 \%$ of the cases [30]. Inverse correlation between VitD status and MS activity was reported.

Runia et al determined in their prospective longitudinal study on 73 patients with relapsing-remitting MS in 2012 that lower serum VitD levels are associated with a higher relapse risk in MS [31].

\section{MS fatigue scores}

Our study suggested that VitD levels in patients with high fatigue score MS were significantly lower than the control group.

Fatigue is considered to be one of the main causes of impaired QoL among MS patients, independent of depression or disability [32]. Fatigue is also among the most common symptoms, reported by at least $75 \%$ of MS patients at some point in the disease course $[33,34]$. Fatigue in MS patients may be multifactorial. In addition to immunologic abnormalities, MS is associated with an increased prevalence of other conditions that contribute to fatigue including depression and several sleep disorders. The most commonly proposed primary mechanisms of fatigue in MS involve the immune system or sequelae from CNS damage. Specific causes are thought to include proinflammatory cytokines, endocrine influences, axonal loss, and altered patterns of cerebral activation [35].

No studies exist in the literature on MS fatigue score and VitD relation. We determined that as VitD level decreases in our patients, fatigue score increases. We think that this can be explained with the depression-decreasing effect of sunlight exposure rather than a decrease in exercise capacity due to limited physical activity, because our patients stated that seeing the sky is the most important therapy that decreases their fatigue and makes them feel good. Our opinion is that making full advantage of direct sunlight exposure especially has a significant positive effect on the treatment.

\section{Conclusion}

As a result, our study indicates that VitD deficiency is a widespread finding in MS and that VitD level increases, and MS year, the number of joint involvement, and spasticity decrease. Accordingly, QoL increases.

Symptomatic therapy of MS is an important part of a comprehensive treatment plan that aims to improve patients' QoL. In the current era of medical progress, several factors have led to the development of guidelines for MS management. Therefore, VitD deficiency should be taken into account in MS and the deficiency should be corrected.

There is continued need for an evidence-based approach supported by high-quality data from controlled clinical trials.

MS is associated with an increase in the treatment of mild and severe VitD deficiency in the QoL of all patients. Serum VitD levels in patients with MS associated with physical and functional results should be examined. 


\section{Financial Disclosure}

The author declares that this study has received no financial support.

\section{Conflict of Interest}

No conflict of interest was declared by the authors.

\section{References}

1. Ozkan B. Nutritional rickets. J Clin Res Pediatr Endocrinol. 2010;2(4):137-143.

2. Mahon BD, Gordon SA, Cruz J, Cosman F, Cantorna MT. Cytokine profile in patients with multiple sclerosis following vitamin D supplementation. J Neuroimmunol. 2003;134(1-2):128-132.

3. Holick MF, Chen TC. Vitamin D deficiency: a worldwide problem with health consequences. Am J Clin Nutr. 2008;87(4):1080S-1086S.

4. Cantorna MT. Vitamin D and its role in immunology: multiple sclerosis, and inflammatory bowel disease. Prog Biophys Mol Biol. 2006;92(1):60-64.

5. Bikle D. Nonclassic actions of vitamin D. J Clin Endocrinol Metab. 2009;94(1):26-34.

6. Bikle DD. Vitamin D: newly discovered actions require reconsideration of physiologic requirements. Trends Endocrinol Metab. 2010;21(6):375-384.

7. Gilroy J. Basic Neurology (3rd ed). Mc Graw-Hill, New York, St. Louis, San Francisco 2000,pp 199-223.

8. Ferrante P. The puzzling natural history of multiple sclerosis: a challenge for the research and care. J Neurovirol. 2000;6(Suppl 2):S1-3.

9. Langer-Gould A, Popat RA, Huang SM, Cobb K, Fontoura $\mathrm{P}$, Gould MK, Nelson LM. Clinical and demographic predictors of long-term disability in patients with relapsing-remitting multiple sclerosis: a systematic review. Arch Neurol. 2006;63(12):1686-1691.

10. Pierrot-Deseilligny C, Souberbielle JC. Is hypovitaminosis D one of the environmental risk factors for multiple sclerosis? Brain. 2010;133(Pt 7):1869-1888.

11. Ascherio A, Marrie RA. Vitamin D in MS: a vitamin for 4 seasons. Neurology. 2012;79(3):208-210.

12. Holick MF. Vitamin D deficiency. N Engl J Med. 2007;357(3):266-281.

13. Munger KL, Zhang SM, O'Reilly E, Hernan MA, Olek MJ, Willett WC, Ascherio A. Vitamin D intake and incidence of multiple sclerosis. Neurology. 2004;62(1):6065.

14. Munger KL, Levin LI, Hollis BW, Howard NS, Ascherio A. Serum 25-hydroxyvitamin D levels and risk of multiple sclerosis. JAMA. 2006;296(23):2832-2838.

15. Salzer J, Hallmans G, Nystrom M, Stenlund H, Wadell G, Sundstrom P. Vitamin D as a protective factor in multiple sclerosis. Neurology. 2012;79(21):2140-2145.

16. Banwell B, Bar-Or A, Arnold DL, Sadovnick D, Naray- anan S, McGowan M, O'Mahony J, et al. Clinical, environmental, and genetic determinants of multiple sclerosis in children with acute demyelination: a prospective national cohort study. Lancet Neurol. 2011;10(5):436-445.

17. Martinelli V, Dalla Costa G, Colombo B, Dalla Libera D, Rubinacci A, Filippi M, Furlan R, et al. Vitamin D levels and risk of multiple sclerosis in patients with clinically isolated syndromes. Mult Scler. 2014;20(2):147-155.

18. Ascherio A, Munger KL, White R, Kochert K, Simon $\mathrm{KC}$, Polman $\mathrm{CH}$, Freedman MS, et al. Vitamin D as an early predictor of multiple sclerosis activity and progression. JAMA Neurol. 2014;71(3):306-314.

19. Thouvenot E, Orsini M, Daures JP, Camu W. Vitamin D is associated with degree of disability in patients with fully ambulatory relapsing-remitting multiple sclerosis. Eur J Neurol. 2015;22(3):564-569.

20. Farsani ZS, Behmanesh M, Sahraian MA. Interleukin-10 but not transforming growth factor-betal gene expression is up-regulated by vitamin D treatment in multiple sclerosis patients. J Neurol Sci. 2015;350(1-2):18-23.

21. Smolders J, Menheere P, Kessels A, Damoiseaux J, Hupperts R. Association of vitamin D metabolite levels with relapse rate and disability in multiple sclerosis. Mult Scler. 2008;14(9):1220-1224.

22. Harandi AA, Shahbeigi S, Pakdaman H, Fereshtehnejad SM, Nikravesh E, Jalilzadeh R. Association of serum $25(\mathrm{OH})$ vitamin D3 concentration with severity of multiple sclerosis. Iran J Neurol. 2012;11(2):54-58.

23. van der Mei IA, Ponsonby AL, Dwyer T, Blizzard L, Taylor BV, Kilpatrick T, Butzkueven H, et al. Vitamin D levels in people with multiple sclerosis and community controls in Tasmania, Australia. J Neurol. 2007;254(5):581-590.

24. Hatamian H, Bidabadi E, Seyed Saadat SM, Saadat NS, Kazemnezhad E, Ramezani H, Bakhshayesh B. Is serum vitamin D levels associated with disability in patients with newly diagnosed multiple sclerosis? Iran J Neurol. 2013;12(2):41-46.

25. Yildiz M, Tettenborn B, Putzki N. Vitamin D levels in Swiss multiple sclerosis patients. Swiss Med Wkly. 2011;141(w13192.

26. Zettl UK, Henze T, Essner U, Flachenecker P. Burden of disease in multiple sclerosis patients with spasticity in Germany: mobility improvement study (Move I). Eur J Health Econ. 2014;15(9):953-966.

27. Berger T. Multiple sclerosis spasticity daily management: retrospective data from Europe. Expert Rev Neurother. 2013;13(3 Suppl 1):3-7.

28. Kheder A, Nair KP. Spasticity: pathophysiology, evaluation and management. Pract Neurol. 2012;12(5):289-298.

29. Spasticity Online Survey Results. 2008. MessmerUccelli M, editor. MS in focus 12, 24-25. Cambridge Publishers Ltd.

30. Beard S, Hunn A, Wight J. Treatments for spasticity and pain in multiple sclerosis: a systematic review. Health Technol Assess. 2003;7(40):iii, ix-X, 1-111.

31. Runia TF, Hop WC, de Rijke YB, Buljevac D, Hintzen RQ. Lower serum vitamin D levels are associated with a higher relapse risk in multiple sclerosis. Neurology. 2012;79(3):261-266. 
32. Krupp LB, Alvarez LA, LaRocca NG, Scheinberg LC. Fatigue in multiple sclerosis. Arch Neurol. 1988;45(4):435437.

33. Krupp L. Fatigue is intrinsic to multiple sclerosis (MS) and is the most commonly reported symptom of the disease. Mult Scler. 2006;12(4):367-368.
34. Lerdal A, Celius EG, Krupp L, Dahl AA. A prospective study of patterns of fatigue in multiple sclerosis. Eur J Neurol. 2007;14(12):1338-1343.

35. Braley TJ, Chervin RD. Fatigue in multiple sclerosis: mechanisms, evaluation, and treatment. Sleep. 2010;33(8):1061-1067. 